\title{
ENTRE EL 40 Y EL 50 EN LA POESIA ARGENTINA
}

\author{
POR \\ CARLOS GIORDANO \\ Università della Calabria
}

I. Cuando leo los poemas de Oliverio Girondo, de Enrique Molina y de Edgar Bayley y pienso en la recíproca comprensión y en la sincera admiración que vinculó a estos poetas, al menos desde $1950^{1}$, me asaltan graves dudas sobre la legitimidad de una tarea que intenta definir y clasificar generaciones o movimientos literarios, casi siempre oponiéndolos entre sí; de una tarea que, negándose aparentemente al goce y la emoción del individual poético, halla su principio en las nociones de proceso y cambio y su método en una cauta generalización.

Estas dudas deberían desaparecer si se postula como la sola tarea significativa la consideración minuciosa de una esencia poética respecto de la cual las obras son concreciones más o menos felices o perfectas. Claro está que así se minimiza la dimensión histórica (con su consecuen-

${ }^{1}$ En la «Cronología» incluida en las Obras completas de Oliverio Girondo (Buenos Aires: Ed. Losada, 1968, pp. 41-43) se señala el año 1946 como la época en que Girondo establece «vínculos más firmes con poetas jóvenes, como Enrique Molina, ... Edgar Bayley...». El 1. ${ }^{\circ}$ de abril de 1951, Girondo publica en el diario La Nación, de Buenos Aires, un largo poema, «Instancias a un poeta encallado en las costas del Pacífico», dedicado a Enrique Molina (recogido en las Obras completas, pp. 396-398). En un libro de poemas de Edgar Bayley, El día (Buenos Aires: Ediciones del Mediodía, 1968), encontramos un poema - «E. M.» (p. 32)que, más que dedicado a Molina, tiene como tema la admirada figura del poeta. En este mismo libro de Bayley figura el conmovido «Homenaje a Oliverio Girondo» (pp. 41-42). Por último, deben recordarse los ensayos de Molina sobre la poesía de Girondo y sobre la de Bayley («Hacia el fuego central o la poesía de Oliverio Girondo» figura como introducción en las Obras completas ya mencionadas, pp. 9-40; «Tanto has andado Edgar Bayley» se publicó en la antología Poesía argentina contemporánea (Buenos Aires: Fundación argentina para la poesía, 1978), tomo I, pp. 124-128. 
te relativismo) y es posible precipitarse en la más abstracta e inmóvil de las metafísicas.

Sea como fuere, el objeto de este breve ensayo de historia de la literatura lo constituye el examen de algunos aspectos de la a veces llamada tercera generación vanguardista ${ }^{2}$ en el panorama general de la poesía argentina del siglo $\mathrm{xx}$. Los aspectos que se tratarán se refieren sobre todo a la tendencia conocida como Invencionismo y el análisis se apoyará preferentemente en algunos textos teóricos.

Como se ve, es un intento muy limitado y aun parcial en su limitación, pero no por eso -es mi esperanza- privado necesariamente de justificación. Se me permita, por último, formular tres breves aclaraciones: Primera, la historia literaria es menos la historia de los hechos puros de literatura (que en sí mismos parecen reclamar la ausencia del tiempo y el espacio) cuanto la historia de una compleja y mezclada institución que se reconoce en el concepto de literatura. Segunda, una historia semejante encuentra su razón de ser no tanto en hechos exteriores que se presentarían como meros agregados a la creación literaria, sino principalmente en la actitud de los creadores mismos cuando - sobre todo en la literatura moderna- se constituyen en grupos, se oponen a otras tendencias, adhieren explícitamente a doctrinas y, en general, teorizan en manifiestos y proclamas. Tercera, en relación con los grupos literarios y las declaraciones teóricas es harto frecuente que la obra individual del artista no corresponda exactamente con estas declaraciones (aunque sea el autor de ellas) y que su vinculación a grupos o tendencias sea notablemente transitoria cuando no conflictiva.

II. Entre 1921 y 1922 se produce un cambio en el proceso de la poesía argentina: se componen grupos innovadores que, al menos en sus declaraciones programáticas, evidencian una actitud decididamente crítica, un verdadero rechazo respecto de la situación literaria contemporánea, es decir, respecto del estilo o los estilos conformados por sus predecesores. En este caso, el modernismo y el sencillismo. Todo esto bajo la influencia o en relación con algunos de los movimientos vanguardistas europeos. Tenemos, pues, el primer movimiento de vanguardia en la Argentina, aunque sería más exacto hablar sólo de la poesía lírica argentina, visto que la narrativa pareciera moverse con tiempos y esquemas diversos. Aun formulado con extrema generalidad -como lo he hecho aquí-, el concepto de esta primera vanguardia poética en

\footnotetext{
${ }^{2}$ Cf. César Fernández Moreno, La realidad y los papeles (Madrid: Ed. Aguilar, 1967), pp. 43 y 320 y ss.
} 
la Argentina constituye hoy una hipótesis suficientemente demostrada y, casi sin excepciones, aceptada y utilizada por críticos e historiadores. No mencionar los nombres de Jorge Luis Borges y de Oliverio Girondo y de las revistas Proa y Martín Fierro sería inexcusable.

Hacia 1940 se produce un nuevo cambio: alrededor de esa fecha es posible hablar de una nueva tendencia en la poesía argentina. Un difuso grupo de poetas, en Buenos Aires y en el interior del país, puede calificarse como neorromántico. Quiero decir que nos encontramos con lo que se considera una nueva generación poética ${ }^{3}$.

César Fernández Moreno es el mayor intérprete e historiador de la generación del $40^{4}$, generación a la que, por otra parte, perteneció como

${ }^{3}$ La actividad «generacional» comienza a manifestarse hacia 1935 y puede considerarse colectiva y relativamente coherente hasta 1945. Tuvo sus revistas: entre las principales, Canto (dirigida por Miguel Angel Gómez, Julio Marsagot y Eduardo Calamaro; dos números en 1940), Huella (dirigida por José María Castiñeira de Dios; dos números en 1941) y Verde memoria (dirigida por Ana María Chouhy Aguirre y Juan Rodolfo Wilcock; seis números entre 1942 y 1944). El caso de la revista $E l 40$ (dirigida por Dora S. de Boneo) es muy particular, pues su primer número es de 1951 y el sexto y último de 1953: se trata de una tardía publicación cuyo objetivo no puede ser otro que el de reconstituir el grupo neorromántico o, por lo menos, el de probar la importancia que éste oportunamente tuvo. Poesía argentina (1940-1949), antología compilada por David Martínez (Buenos Aires: Col. El Ciervo en el Arroyo, 1949), puede ser considerada la selección más representativa del neorromanticismo cuarentista. Revistas, antologías y libros nos proporcionan los nombres de más de setenta poetas, típicos representantes del neorromanticismo en esos años o vinculados a esta tendencia. Sería injusto nombrar algunos y callar el nombre de la mayoría. Sin embargo, César Fernández Moreno elige seis nombres para ilustrar las cinco tendencias en que divide el movimiento: Vicente Barbieri, Juan G. Ferreira Basso, Miguel Etchebarne, Juan Rodolfo Wilcock, Daniel Devoto, Enrique Molina (op. cit., p. 239). El tremebundo Eduardo Romano deja en pie, aunque muy vapuleados, sólo a Eduardo J. Bosco, Jorge Calvetti y Enrique Molina. Muty significativa es la inclusión de Homero Manzi, «el mayor poeta - según Romano- de esos años difíciles» (Eduardo Romano, "Qué es eso de una generación del 40», ensayo incluido en Romano, Becco, Giordano, El 40, Buenos Aires: Editores Dos, 1969, pp. 39-91). En cuanto a manifiestos «generacionales», merecen recordarse las declaraciones preliminares de los dos números de Canto y del primer número de Verde memoria, y la «Carta abierta: 'Canto' se define y nos define», de Carlos Alberto Alvarez (en Argentina Libre, núm. 20, Buenos Aires, 18 de julio de 1940). De su lectura es posible concluir que el neorromanticismo no fue demasiado agresivo con sus predecesores y que, en cuanto a formular un programa, se movió en términos de extrema vaguedad.

${ }^{4}$ Desde «Informe sobre la nueva poesía argentina» (en Nosotros, 2. época, año VIII, núm. 91, Buenos Aires, octubre de 1943, pp. 71-93) hasta el libro ya mencionado, La realidad y los papeles (especialmente los capítulos II, X, XI, XII y XIII), pasando por «La poesía argentina de vanguardia», su contribución a la 
poeta. Ahora bien: el método histórico de las generaciones ha sido aplicado con sorprendente entusiasmo por muchos de los historiadores y antólogos de la literatura argentina (en Fernández Moreno constituye el rígido núcleo de su metodología), pero con resultados que, a mi juicio, no compensan con la eventual claridad de sus ordenamientos el esquematismo y el mecanicismo implícitos en el método mismo. Como alguna vez he señalado ${ }^{5}$, yo no creo en la utilidad del método histórico de las generaciones y prefiero manejarme con las categorías de estilo, tendencia, movimiento, escuela o grupo, según convenga. Por otra parte, no es ésta una discusión sobre la que valga la pena detenerse demasiado, y si aquí la cuestión ha sido mencionada es porque justamente una de las primeras cosas que llama la atención en las declaraciones de los propios protagonistas y desde el inicio del movimiento neorromántico es su preocupación sobre la circunstancia de si constituían o no una generación literaria. Este hecho puede interpretarse de muy diversos modos y atribuírsele mayor o menor importancia. Para mí, es la prueba «al revés» del extremo individualismo del que proviene la actitud y la concepción estética neorromántica, individualismo que si bien es ostentado orgullosamente como uno de los más altos valores humanos y del poeta en particular, es al mismo tiempo sentido como irremediable soledad, como una trágica condición que trata de paliarse, tal vez sin advertirlo, por vías secundarias y escondidas. Este exacerbado individualismo es, a su vez, una de las respuestas posibles frente a la crisis, frente a lo que se ve sólo como el convulsionado desintegrarse de un orden que por ello mismo se absolutiza, en el sentido de que a su destrucción no puede seguir sino el caos o la nada.

Un análisis causal sociológico de la literatura argentina entre los años 1930 y 1950 deberá atender a la relación entre los productos lite-

Historia de la literatura argentina, dirigida por Rafael Alberto Arrieta (Buenos Aires: Ed. Peuser, 1959, tomo IV, pp. 605-669). Todo ello entre un gran número de otros artículos y libros en los que analiza y valora el neorromanticismo.

${ }^{5}$ «Temas y direcciones fundamentales de la promoción poética del 40 » (en Boletín de literaturas hispánicas, Facultad de Filosofía y Letras, Universidad Nacional del Litoral, núm. 5, Rosario de Santa Fe, Argentina, 1963). Todas las consideraciones que seguirán sobre la tendencia neorromántica en la poesía argentina reenvían a este ensayo de 1963 y deben confrontarse con los capítulos ya señalados, en la nota precedente, de La realidad y los papeles, de César Fernández Moreno. Después de casi veinte años, obviamente no coincido con todo lo que entonces dijera, aunque suscribo todavía las tesis fundamentales de mi ensayo. Ello me coloca otra vez en la situación de disentir amablemente con Fernández Moreno. Nuestras interpretaciones difieren en aspectos no secundarios, lo cual no implica que no estemos de acuerdo en muchos otros. 
rarios y determinados hechos históricos, pero sobre todo a una atmósfera creada por estos hechos y por sus consecuencias y por los presagios de nuevos eventos posibles. Esta atmósfera es en sí una interpretación de la historia, objeto de la sociología en cuanto generalizada y representativa de un grupo social. De todos modos, las circunstancias históricas no condicionan a priori y con carácter de necesidad el tipo de reacciones que ante ellas es inevitable adoptar, así como la posterior valorización que se haga de estas reacciones depende de la perspectiva ideológica del crítico o del historiador.

E1 golpe militar de Uriburu en 1930; el período que le sigue (la llamada "década infame»), con el progresivo agudizarse de las contradicciones económicas, sociales y políticas -período que significa el fracaso de la derecha nacionalista, pero que también cierra todas las posibilidades a las izquierdas tradicionales-; el posterior golpe militar de 1943, con sus asomos de fascismo; los movimientos populares de 1945 y la profunda modificación que significó la primera presidencia de Perón y la obra de Eva Duarte desde la Secretaría de Trabajo y Previsión; las nuevas contradicciones que se generan en el régimen peronista como resultado de su creciente burocratización y que tratan de ocultarse con un pesado sistema de propaganda y una relativa represión política. Todo ello visto y sentido desde la ideología liberal democrática a la que está soldada firmemente la mayor parte de la intelectualidad argentina, sea en lo que, para entendernos, podemos llamar su ala derecha, sea en su ala izquierda. Debemos agregar una situación internacional tal que nos exime de mayores comentarios: gobiernos dictatoriales en Europa, guerra de España (nunca se insistirá demasiado sobre las consecuencias de este conflicto entre los escritores argentinos), invasión de Polonia por parte de Alemania y Rusia e inicio de la segunda guerra mundial; fin de la guerra con tremendas comprobaciones y funestos augurios. Frente a un panorama semejante, las respuestas posibles son múltiples y de hecho lo fueron ${ }^{6}$. El joven neorromanticismo es una de ellas.

Del individualismo ya señalado (a partir de una circunstancia marginal) no es difícil pasar a la consideración del extremo subjetivismo de

${ }^{6}$ Es interesante recordar que después de 1930 muchos martinfierristas cambiaron o, tal vez, radicalizaron su posición: Raúl González Tuñón hacia el partido comunista, Leopoldo Marechal hacia la extrema derecha, Borges hacia la literatura fantástica. Con la guerra de España, Córdova Iturburu va hacia la izquierda. Con el peronismo al poder, algunos cuarentistas pasan a sus filas: José María Castiñeira de Dios y Miguel Angel Gómez, por ejemplo. Resta el problema de valorar hasta qué punto los cambios de posición política comportaron cambios en el estilo literario. 
los poetas del 40 , pero la subjetividad -y esto es verdaderamente definitorio- es concebida sobre todo (casi exclusivamente) como puro sentimiento. Individualidad, subjetividad y sentimentalidad son equivalentes y forman un todo indisoluble. En este orden de cosas, el poeta y la poesía devienen entidades privilegiadas. El poeta, porque frente a la inminencia del caos es el único que, a través de su intensísimo sentimiento, sostiene los perennes valores ahora amenazados. La poesía, porque así se le otorga una función casi redentora. No obstante, sería un grave error menospreciar la denuncia explícita que una tal concepción comporta, así como la implícita aspiración a un mundo diferente. Los neorománticos, sin embargo, acentuaron en el conflicto entre subjetivo y objetivo un profundo sentimiento de fracaso. La desgracia es una experiencia que otorga a la condición humana su más alto valor, sólo en ella el sentir alcanza verdadera intensidad, es sólo ella la que permite distinguir entre elegidos y réprobos. Véase como ejemplo de ello la tratación que esta literatura hace del amor: el objeto de la pasión vale en cuanto perdido o inalcanzable; el goce de su posesión, en caso de darse, se anula en la certeza de su fugacidad. Con todo, no es la desesperación la inevitable consecuencia. Ello se opondría parcialmente a la valorización del poeta y la poesía sostenida en otro nivel. De manera muy general y sin olvidar las numerosas excepciones, los neorrománticos argentinos optaron por una especial forma de nostalgia. Los grandes valores (la felicidad) existieron en una edad dorada, ahora definitivamente perdida. De allí su especial visión del amor, de la infancia y de la patria. De allí su tono con frecuencia elegíaco.

La patria, el sentimiento de la patria, fue uno de los temas importantes de los poetas del 40. Fernández Moreno piensa que en ellos «el canto de lo argentino resulta más tangible y carnal...». Con lo argentino - como con cualquier otra presunta forma de la realidad - todo depende de lo que entendamos como tal. A mí me parece que los neorománticos privilegiaron la naturaleza - concebida de una manera que resultase coherente con el trascendentalismo de su poesía- y el paisaje, en cuanto vehículos para la expresión de sus sentimientos. Este mecanismo a través del cual aparece la geografía patria no es nuevo y constituye un legítimo recurso; más dudosa me parece su insistente pretensión de trascendentalidad. En el caso de la historia, una gesta mítica denuncia tácitamente desde el ayer la traición del presente a un prefigurado destino. Esta poesía rescata y propone así un abstracto ser nacional -más esencia que existencia-, un ser que se define en la evocación del pasado irrecuperable o en la melancólica soledad ante lo telúrico. 
Por otra parte, el neorromanticismo argentino enfrentó el problema de la comunicabilidad de la poesía, y por esa vía el problema de la forma poética. Se trataría de una reacción contra el hermetismo de la vanguardia precedente. No puedo entender este problema sino en relación con la modesta destrucción formal que practicaron algunos ultraísmos. La estética cuarentista configuraría una poética de la expresión y no de la construcción, para decirlo brevemente. En consecuencia, una poética que mira a la comunicación como su presupuesto básico. Pero es aquí que los poetas del 40 reiteraron una solución que no por conocida es menos efectiva. Una solución que, en los casos más logrados, nos ofrece una poesía de gran calidad y que por ello alcanza a superar la cuestión del acuerdo que podemos prestar o no a la actitud central de que proviene. Todo esto si se acepta que no es condición necesaria y suficiente a la poesía un tipo de comunicación similar o comparable a la comunicación pragmática y cotidiana. La solución de que hablo es el equilibrio entre una no destrucción de las estructuras lógicas del lenguaje y la transformación de ese lenguaje mismo por obra del complejo sistema de la versificación tradicional utilizado con sorprendente habilidad. Hay, pues, una clara voluntad constructiva de un cierto tipo. El peligro de semejante estilo es que puede no exceder la trivial imitación arcaizante, así como degenerar en fácil «maniera», por una parte, mientras por otra es siempre proclive a la afectación elitista. $Y$, en efecto, algo de eso ocurrió con demasiada frecuencia entre los poetas del 40, aunque al mismo tiempo nos ofrezcan algunos poemas de perdurable belleza. Hablo de poemas y no de ocasionales versos felices.

Soslayaré el problema de las influencias, pues él implica menos un catálogo de nombres y la comprobación del influjo que ejercieron que el cuidadoso análisis de cómo fueron interpretados por los influidos los autores a que se refieren las influencias. En el caso que nos ocupa se habla siempre de Rilke, de Neruda y de la generación española de 1927, sin olvidar —obvio es decirlo- a los grandes románticos europeos.

No sabría decir si los poetas del 40 fueron «hipervitales» o "hiperartísticos», pero tampoco siento la necesidad de decidirme ante esta disyuntiva. Pienso que sus características principales justifican que se los interprete y califique, en conjunto, como neorrománticos. No estoy en absoluto de acuerdo con que se hable de ellos como de la segunda generación de vanguardia. Significaron, más bien, una interrupción del proceso iniciado por lo que hubo de vanguardismo en los grupos literarios de los años veinte. Lo cual no es, necesariamente, un juicio de valor negativo. El proceso de la vanguardia se reiniciará, alrededor de 1950, tal vez (como es mi hipótesis) enriquecido por la experiencia neorro- 
mántica. El neorromanticismo forma parte - como tantas otras escuelas- de lo que podemos llamar literatura moderna, pero no puede confundirse con un movimiento de vanguardia: entre muchas otras cosas, por sus opuestas concepciones del autor, de la obra y de la actividad literaria. Neorromanticismo y vanguardia son nociones que se excluyen recíprocamente ${ }^{7}$.

III. En la contratapa del único número de la revista Arturo (Buenos Aires, verano de 1944) leemos esta sintética declaración: «Júbilo. Negación de toda melancolía. Voluntad constructiva. Comunión. Poesía del contrato social.» Nos encontramos con conceptos que, en relación con el neorromanticismo de tres o cuatro años antes, no pueden interpretarse sino como el signo de una voluntad de cambio. La poesía es concebida ahora como todo lo contrario de lo que sostuvieron los poetas del 40: a la melancolía se opone el júbilo, a la expresión la construcción, a la soledad la comunión (precisada esta última con referencia a lo social). Con Arturo ha comenzado a manifestarse lo que César Fernández Moreno llama la «tercera generación vanguardista» ${ }^{8}$ y yo preferiría considerar como la segunda o, más precisamente, como la aparición en esa época de una tendencia neovanguardista en la poesía argentina. Por otra parte, este neovanguardismo - como es característico en los movimientos de vanguardia - no se limitará a la literatura, sino que planteará la cuestión en términos de la relación entre las artes: una nueva tendencia en la poesía y una nueva tendencia en la plástica, a partir de problemas comunes y con soluciones equivalentes. Para decirlo

${ }^{7}$ Deliberadamente he dejado de lado el problema del surrealismo en su relación con la poesía de los neorrománticos argentinos. Ello es grave porque Enrique Molina es, quizá, el poeta más significativo del cuarenta y porque, además, la influencia de Pablo Neruda (sobre todo el de Residencia en la tierra) es evidente en muchos de ellos. Sucede que la mera aproximación al tema excedería en mucho los límites de este artículo. Señalaré, sin embargo, que - a mi juicio- se ha abusado en la atribución de la calidad de surrealista. El surrealismo es un movimiento más preciso en su estética y en su poética de lo que a veces se sostiene. Claro está que rasgos surrealistas se pueden encontrar con mucha frecuencia y en las más diversas combinaciones (cf. Graciela de Sola, Proyecciones del surrealismo en la literatura argentina (Buenos Aires: Edic. Culturales Argentinas, 1967).

${ }^{8} \mathrm{Cf}$. La realidad y los papeles, p. 43 . La cronología generacional debe manejarse ahora con lapsos demasiado estrechos: César Rosales admite sólo la generación del 40 y lo que le sigue no es sino su consecuencia; Emir Rodríguez Monegal, para reunir gran parte del 40 y del 50, propone una del 45, apoyándose en un acontecimiento exterior condicionante: el peronismo; Fernández Moreno habla de dos generaciones, pero «integrativas» (op. cit., pp. 321-322). 
con simpleza, invencionismo en la poesía (Edgar Bayley), artes visuales no figurativas o abstractas (Tomás Maldonado) ${ }^{9}$.

En 1945 se publican los cuadernos Invención y en 1946 el Boletín de la Asociación de Arte Concreto-Invención. También en 1946 se produce una primera escisión: Gyula Kosice y Carmelo Arden Quin forman un nuevo grupo, madí (materialismo dialéctico), para preservar o extremar el rigor de los principios originarios del movimiento. En 1949 es la revista Contemporánea (dirigida por Juan Jacobo Bajarlía) la publicación más representativa del invencionismo poético. Poesía Buenos Aires, que comienza a publicarse en 1950 (dirigida por Raúl Gustavo Aguirre y también ocasionalmente por Jorge Enrique Móbili, Volf Roitman, Nicolás Espiro y Edgar Bayley), constituirá «la más perdurable de las revistas exclusivamente poéticas editadas en cualquier tiempo en la Argentina» ${ }^{10}$.

Las dos alas en que se dividiría el movimiento de innovación de la promoción literaria de 1950 (llamémosla así para entendernos) serían la del ya mencionado invencionismo y la de un surrealismo practicado y teorizado con rigor y hasta con cierta intransigencia. En 1952 aparece $A$ partir de cero, "revista de poesía y antipoesía», dirigida por Enrique Molina, y entre 1953 y 1954 se publica Letra y Línea, bajo la dirección de Aldo Pellegrini. La presencia de Molina (poeta que proviene del 40) y el hecho de que Pellegrini fundara el primer grupo surrealista en la Argentina en 1928 (su revista, Que, publicó dos números en 1928 y en 1930) son circunstancias muy significativas, aunque no autoricen a sostener que una constante surrealista caracteriza la poesía moderna rioplatense.

La antología de Horacio Jorge Becco y Osvaldo Svanascini, Poesía argentina moderna (Buenos Aires: Ediciones Pedestal, 1953) y la de Raúl Gustavo Aguirre, Antología de una poesía nueva (Buenos Aires: Ediciones Poesía Buenos Aires, 1952), pueden considerarse las dos selecciones poéticas más representativas del neovanguardismo. La antología de Aguirre y el número 13/14 de Poesía Buenos Aires (1954), dedicado a una "Imagen de la nueva poesía», evidencian no sólo la evolución sufrida por el propio invencionismo, sino también el esfuerzo de esta

9 El término abstracto, aplicado al arte en oposición a figurativo, crea un serio problema a quienes rechazan, en su concepción del mundo, la abstracción metafísica para acercarse a una filosofía materialista. Por ello, abstracto debe entenderse, como luego veremos, en el sentido de concreto. En otro orden de cosas, debe recordarse la preocupación de Bayley por el teatro: en 1951 estrena una farsa, Burla de primavera, en 1952 funda el Teatro Contemporáneo y en 1953 da a conocer su obra teatral Dulioto.

${ }^{10}$ César Fernández Moreno, op. cit., p. 324. 
revista - si se considera su punto de partida invencionista- por representar sin demasiadas tensiones las dos alas innovadoras del 50.

En 1945, en el segundo cuaderno Invención, Edgar Bayley firma un manifiesto titulado «La batalla por la invención». Debe considerárselo el manifiesto del invencionismo literario y la exposición más completa de sus doctrinas iniciales ${ }^{11}$. No es un texto fácil o, para decirlo con más precisión, no lo es en el sentido de que bajo una forma apodíctica y un tono algo presuntuoso, en realidad afronta con lucidez y modestia casi todos los extremos de lo que podríamos llamar la problematicidad de la expresión poética.

Bayley opone el Invencionismo a lo que indistintamente llama Arte Figurativo o Arte Representativo (estas y todas las mayúsculas que siguen le pertenecen), y lo hace en el campo de la relación del creador con la obra y de la obra con el público, pero apoyando todo su razonamiento en la crítica a una segunda relación que así deviene primera y fundamental: la del autor, la obra y el público con la realidad objetiva, entendida más como historia que como naturaleza. Ello explica la sorprendente preocupación ética que demuestra el manifiesto.

El Arte Figurativo está fundado en «la mística del individuo», y para el individualista to fundamental es ser diferente. La diferencia, sin embargo, es un aparente valor que se define menos por sí mismo que por el juicio de los otros. Ello lleva a la necesidad de traducir o expresar a través del arte esa individualidad diferente. Intento inútil, pues «los actos vitales... en cuanto experiencias prácticas no pueden ser reemplazados por ningún signo...». Es éste el arte de la separación, y «separarse es pensar contradictoriamente, es seguir y concebir la vida como una dualidad. La separación exige la expresión, busca o presta a cada cosa una significación antojadiza que tiende a debilitar el poder del hombre sobre el mundo». Así, la oposición entre lo subjetivo y lo objetivo resulta irreductible, pues está mediada falsamente por la representación artística, que no es sino inauténtica deformación de lo uno y lo otro. En lo histórico, el Arte Viejo está formado por los «hijos tardíos del romanticismo» y por «la manía simbolista». El Invencionismo no quiere «representar realidades ni surrealidades» (el subrayado es mío), no quiere «publicar biografías ni autobiografías», niega enérgicamente toda melancolía y «tiende a liberar al Hombre de esos dos frutos de la soledad: el resentimiento y la vanidad».

${ }^{11}$ Cito este manifiesto según el texto reproducido en La realidad y los papeles, pp. 548-549. 
Por un momento nos parece que al invencionismo le bastara que la subjetividad sea reconocida en la propia objetividad y la realidad objetiva aceptada como tal. Pero no es así. La poesía tiene una función específica: «inventar objetos concretos de arte que participen de la vida cotidiana de los hombres, que coadyuven en la tarea de establecer relaciones directas con las cosas que deseamos modificar». Es decir, se propone un arte cuya finalidad no es representativa ni expresiva y que en este sentido está desprovisto de significación, a la vez que en sí mismo es un objeto concreto. Sólo de esta manera, en este extremo objetivismo, se recompone la unidad del mundo y se reconoce a la poesía una tarea también concreta: ayudar a reconstruir el mundo. Una empresa ahora posible por la inmediatez de su participación y legítima por su renuncia a las jerarquizaciones deformadoras de la interpretación.

En el arte invencionista, los «valores de comunión sustituyen a los valores de diferencia» del Arte Viejo. La comunicabilidad artística es posible únicamente a nivel de esta comunión. «La Invención Concreta está infinitamente más cerca de los hombres que cualquier Arte Figurativo.» $\mathrm{Y}$ «estar cerca de los hombres es conquistar para sí la plenitud creadora, el júbilo intraducible e irreemplazable». En cuanto al artista, «no tiene un reino aparte de la realidad común» y «el Nuevo Arte... no es la opinión de un hombre aislado, es una realidad en sí mismo, que no hace pensar en nada ni se parece a nada». El poeta afronta así su responsabilidad «en consonancia con el advenimiento de nuevas formas de organización social y con el progreso de las fuerzas productivas». Como se ve, el manifiesto invencionista se estructura con coherencia, pero con una extrema generalidad conceptual. Se mueve en el ámbito de la pura teoría estética sin intentar formular siquiera la menor norma de poética. Su carácter vanguardista, por otra parte, es evidente. El invencionismo recoge la herencia del creacionismo, al menos en el doble aspecto de evitar la sujeción referencial y de propugnar una actividad artificial (material y concreta) que «non implica una coscienza personalizzata che vuole autoesprimersi» ${ }^{12}$. Acentúa, en cambio, la consideración de las responsabilidades éticas del poeta y el problema de la comu-

${ }^{12}$ Saúl Yurkievich, «Avanguardia latinoamericana: rottura della permanenza e permanenza della rottura», en Storia di una iniquità, a cura di Pier Luigi Crovetto (Genova: Tilgher Editrice, 1981, p. 349). Para una ubicación correcta del invencionismo y de Huidobro en el panorama de las vanguardias latinoamericanas, ef. el ya señalado artículo de Yurkievich, de una agudeza crítica a la que mucho debo, y el ensayo de Ramón Xirau "Crise du réalisme" (en L'Amérique latine dans sa littérature, Paris: Unesco, 1979). 
nicabilidad de la poesía. El «pequeño dios» de Huidobro se democratiza ${ }^{13}$.

En 1952, Bayley publica un ensayo que de alguna manera completa el manifiesto de 1945, en cuanto ahora se insinúan algunas líneas generales de poética. Desde otro punto de vista, el texto de 1952 significa una integración más amplia de la estética invencionista por la aceptación de elementos que provienen del romanticismo y del surrealismo (¿por influencia de la poesía del 40?), aunque estos movimientos -en su conjunto- sean objeto de muchas reservas. Se trata de Realidad interna y función de la poesía (Buenos Aires: Ediciones Poesía Buenos Aires, 1952, 35 págs.).

La «realidad interna» de la poesía se identifica ahora claramente con el lenguaje poético, lenguaje inhabitual cuya validez depende de «la combinación inventiva de las palabras». Por eso objetará a la imagen surrealista que «la invención poética reside, más que en la relación verbal, en el carácter de las situaciones descritas», y a la imagen creacionista que «permanece aún dentro de la mecánica imitativa», aunque en teoría haya afrontado el problema de las «relaciones de palabra a palabra».

En el manifiesto de 1945 se subrayaba la naturaleza innovadora del invencionismo, refiriéndose sin mayores precisaciones a una batalla comenzada a principios de este siglo. Ahora se sostiene, en cambio, que la condición inventiva del poema ha sido siempre la característica de toda poesía verdadera. La época moderna no habría sino reconocido la importancia de la invención hasta transformarla «en el principal fundamento de una teoría y una práctica poéticas». Y, en verdad, la mayor parte del ensayo de Bayley está dedicada, desde este punto de vista, a analizar con gran agudeza ejemplos poéticos, desde los trovadores provenzales a nuestros días. De los análisis textuales que realiza es posible

${ }^{13}$ Esta negación de la melancolía y este júbilo de la creación que caracterizan los inicios del invencionismo, ¿de dónde provienen en el orden de las causas histórico-sociológicas? Aventuro la hipótesis de que su origen debe buscarse en el final de la segunda guerra mundial, con la derrota del fascismo y el triunfo de los aliados; pero, sobre todo, en el hecho de que entre los vencedores se encuentra el socialismo ruso. No tengo elementos para imaginar por ahora que el período que media entre el golpe militar de 1943 y el 17 de octubre de 1945 permitiera a una parte de la intelectualidad argentina concebir de inmediato alegres esperanzas sobre el futuro del país. Pero también es posible. Otra hipótesis consistiría en suponer que el júbilo invencionista es el resultado de una heroica voluntad que, frente a la crisis general de Occidente, reacciona en sentido diametralmente opuesto al de los neorrománticos. 
inferir ciertas normas de poética que completan el principio básico de la combinación verbal.

La novedad más importante que introduce este segundo texto consiste, primero, en la aceptación de que la experiencia emocional es «imprescindible para la existencia misma de la poesía», no sólo como principio motor del acto de escribir, sino también en cuanto «concurre a dar una coherencia de fondo al poema». No obstante, el poema «inventa y no traduce», porque cuando el poeta comienza a escribir «se produce lo que René Char llama la elección objetiva». En segundo lugar, la elección objetiva de las palabras y su combinación inventiva se hace sobre la base de lo que Bayley llama el «tono» o «las valencias poéticas de las palabras», y que podría interpretarse, creo yo, como una referencia a los valores connotativos del lenguaje. Es fácil deducir que para Bayley la operación poética, a pesar de la intervención del sentimiento, es guiada, sobre todo, por la inteligencia $o$, al menos, por un especial tipo de lucidez cuya característica específica sería su libertad operativa.

La «función poética», por su parte, es una «función normal del espíritu humano» y «no tiene carácter excepcional». El acto poético se cumple para satisfacer una necesidad humana: relacionarse con las cosas y dominarlas. La poesía sirve para que el hombre mantenga «tensa una disposición de su espíritu, sin la cual no habría podido construir el mundo que le rodea, edificándose, por añadidura, a sí mismo». En idéntico orden de cosas se mueve con un propósito de integración social.

Es la posesión, común a todos los hombres, de la «disposición poética» lo que garantiza la comunicabilidad del poema. Es la sensibilidad - nunca confundida con la mera sentimentalidad - lo que permite la comprensión del objeto poético inventado. Ocasionales dificultades en este campo no dependerían sino de «hábitos perceptivos inadecuados».

Entre 1945 y 1952 el invencionismo literario produjo obra poética (válida, en especial, la del autor de los dos textos analizados) y no creo que en ella se traicionaran los principios del movimiento. Creo simplemente que esa experiencia obligó a ampliarlos. Dejando aparte la diferente calidad de ambos (uno es un ensayo y el otro un manifiesto), el primero evidencia un extremo rigor intelectual y obvias limitaciones (no desciende al nivel de una poética operacional, afirma como válidos sólo los principios que enuncia sin intentar siempre probarlos), mientras el segundo gana en amplitud, pero pierde algo en rigor (apela reiteradamente a nociones como las de sensibilidad y espíritu, fundamenta la creación y la comunicación poéticas en niveles que parecieran vacilar a veces entre el orden de la inteligencia y el de la emotividad). No sostengo que sea así tout court, no estoy poniendo en tela de juicio, tam- 
poco, la notable significación de cada uno de estos textos, estoy tratando de afirmar que se complementan de una manera notable sin contradecirse en los principios fundamentales. Referido el uno al otro, limitaciones y debilidades se atenúan. Una adecuada comprensión del movimiento invencionista en la Argentina ${ }^{14}$ exige esta lectura conjunta, y pocos son los movimientos que supieron estructurar una teoría de esa coherencia. Discutir la calidad de la poesía invencionista no invalidaría la afirmación precedente.

Casi al final de su ensayo de 1952 Bayley dice: «He querido poner el espíritu crítico al servicio de la inocencia.» Yo quisiera terminar estas reflexiones con las palabras iniciales de un poema de su primer libro: «La poesía es invadida por momentos, pero la resistencia existe, no lo dudemos. Esa resistencia está en las calles, a horas determinadas, en las conversaciones, en el cielo de cada mano.» Es esta resistencia una circunstancia promisoria, aunque los invasores sean el propio Bayley o yo mismo.

${ }^{14} \mathrm{El}$ invencionismo es, por sus propios principios, contrario a la expresión de todo nacionalismo literario, pero ello no excluye que en su materia poética no sean muy visibles formas típicas de una manera de ser nacional. Recuérdese su insistencia en la cotidianeidad y el hecho básico de que sea el «tono» de las palabras lo que interesa especialmente a su poética. 